TECHNICAL TRANSACTIONS 1/2017

CZASOPISMO TECHNICZNE $1 / 2017$

PHYSICS

DOI: $10.4467 / 2353737$ XCT.17.015.6112

\begin{abstract}
Andrzej Osak (aosak@ifpk.pk.edu.pl)
Institute of Physics, Faculty of Physics Mathematics and Computer Science,

Cracow University of Technology
\end{abstract}

\title{
AC LOW FREQUENCY CONDUCTIVITY IN PZT PFS FERROELECTRIC
}

\section{CERAMICS}

NisKOCZĘSTOTLIWOŚCIOWE PRZEWODNICTWO ELEKTRYCZNE
FERROELEKTRYCZNEJ CERAMIKI PZT PFS

\begin{abstract}
Low frequency AC conductivity has been studied in ferroelectric ceramics PZT $+\mathrm{PFS}\left(\mathrm{Pb}\left[\left(\mathrm{Fe}_{1 / 3} \mathrm{Sb}_{2 / 3}\right) \mathrm{Ti}_{x} \mathrm{Zr}\right] \mathrm{O}_{3}\right.$ with $x=0.1$ and $y=0.43,0.44,0.47$ ) using Fourier transformation of charging and discharging currents. The results are interpreted in terms of fractal structure of the randomly generated clusters formed by sequentially correlated hopping paths of charge carriers.
\end{abstract}

Keywords: PZT, AC conductivity, fractal structure conduction clusters

\section{Streszczenie}

$\mathrm{W}$ artykule przedstawiono przewodnictwo zmiennoprądowe ceramiki ferroelektrycznej PZT + PFS $\left(\mathrm{Pb}\left[\left(\mathrm{Fe}_{1 / 3} \mathrm{Sb}_{2 / 3}\right)_{x} \mathrm{Ti}_{y} \mathrm{Zr}_{z}\right] \mathrm{O}_{3}\right.$, gdzie $x=0.1$ i $\left.y=0,43,0,44,0,47\right)$ w zakresie ultra niskich częstotliwości obliczone na podstawie analizy Fouriera prądów polaryzacji i depolaryzacji dielektrycznej. Wyniki zostaty zinterpretowane na podstawie fraktalnej struktury klasterów generowanych przez stochastyczny sekwencyjny hopping nośników ładunku.

Słowa kluczowe: PZT, przewodnictwo zmiennoprądowe, fraktalna struktura przewodzących klasterów 


\section{Introduction}

The investigation of dielectric and electric properties in disordered solids has been the subject of a great deal of interest because of their many technical applications.

From the phase diagram of the PZT $\left(\mathrm{Pb}\left(\mathrm{Zr}_{1-x} \mathrm{Ti}_{x}\right) \mathrm{O}_{3}\right)$ ferroelectric solid solution follows that in this compound the phase boundary exists which divides regions with rhombohedral and tetragonal structures [1,2]. Recently Noheda et al. [3] reported that, within a narrow region $0.45<x<52$, there was a bringing monoclinic phase. This region is called the morphotropic phase boundary (MPB). It was established that in the MPB there is a progression from long range to short range structural orders leading to a stochastic disorder [4]. In the studied complex compound $\mathrm{PZT}+\mathrm{PFS}\left(\mathrm{Pb}\left[\left(\mathrm{Fe}_{1 / 3} \mathrm{Sb}_{2 / 3}\right)_{x} \mathrm{Ti}_{y} \mathrm{Zr}_{z}\right] \mathrm{O}_{3}\right.$ with $x+y+z=1, x=0.1$ and $y=0.43,0.44,0.47)$ the crystal lattice $\mathrm{Pb}$ sites are partially replaced by $\mathrm{Sb}$ cations while the Ti sites by $\mathrm{Zr}$ or Fe cations. The structural studies performed for the 0.43 and 0.44 of Ti content samples established the existence of the MPB region [5]. The coexistence of rhomboedral and tetragonal phases in the MPB range as well as doping leads to structural disorder in the studied compound.

AC conductivity measurements are an important means to study deep defect centres. The alternating current (AC) conductivity of disordered solids shows a dispersive behaviour through the dependence of electrical conductivity $\sigma(\omega)$ vs. frequency $\omega$ of the form [6].

$$
\sigma(\omega)=\sigma_{d c}+\sigma_{p}(\omega)
$$

where $\sigma_{d c}$ is the direct current (DC) conductivity and $\sigma_{p}(\omega)$ is the frequency dependent polarization conductivity. Polarization conductivity $\sigma_{p}(\omega)$ is created due to the relaxation process connected with defect centres or group of centres that develop electric dipole moments under the action of applied electric field. A strong frequency dispersion of AC conductivity follows from a broad distribution of the relaxation times [7]. The measurement of the step response function over several decades of time (up to $10^{4} \mathrm{~s}$ ) is usually used to investigate low frequency AC dielectric losses. The dielectric response in the frequency domain is described in terms of dielectric susceptibility $\chi(\omega)$ or permittivity $\varepsilon(\omega)$ functions. The dielectric properties can be equivalently expressed in terms of complex conductivity.

$$
\sigma(\omega)=-i \omega \chi(\omega)
$$

In order to convert results from the time- into frequency domain the one-side Fourier transform is commonly used.

The AC conductivity $\sigma(\omega)$ in disordered solids depends strongly on the external field frequency. At high frequency range, one observes a sublinear dependence $\sigma(\omega) \propto \omega^{s}$ with $0<s<1$, whereas at low frequency range a supralinear dependence with the power exponent $s>1$. The cross-over from high-to low frequency range takes place at the dielectric loss peak frequency $\omega_{c}$. 
The AC conductivity in disordered solids has been studied in terms of hopping mechanism of charge carriers between localization sites. Many models based on relaxation caused by hopping or tunnelling of electrons, polarons or ions between equilibrium states, have been proposed [8-15] to explain the frequency and temperature dependencies of AC conductivity.

\section{Theory}

\subsection{Rate equitation}

In disordered solids the electron hopping process takes place between localized states that are statistically distributed in space and in energy. According to Miller and Abrahams [9], the electron transition probability $w_{i j}$ between the localized states $r_{i}$ and $r_{j}$ depends on the tunnelling probability, proportional to $\exp \left(-2 a r_{i j}\right)$ and the energy difference between both sites $E_{i j}=E_{i}-E_{j}$ taking the following form

$$
w_{i j}=w_{0} \exp \left(-2 a r_{i j}-E_{i j} / k T\right)
$$

In this expression $k$ is the Boltzmann contrast, $T$ is temperature and $a$ is localization length of the wave function $\psi$ in a site $r_{i}$, i.e. $\psi \propto\left[-\left|r-r_{i}\right| / a\right]$. The transition rate is defined as the product of the site transition probability $w_{i j^{\prime}}$ occupation probability of the initial site $P_{i}(t)$ and non occupation probability of the final state $w_{i j} f_{i}\left(1-f_{j}\right)$, where $f$ is the Fermi function. The transition rate fulfils the following master equation

$$
\frac{d P_{i}(t)}{d t}=\Sigma_{j} w_{j i} P_{i}(t)-\Sigma_{i} w_{j i} P_{i}(t)
$$

In order to determine the conductivity we must consider the transition rate in the presence of an electric field. The electric field changes the occupation probability $P(t)$ of localized states. In the linear approximation (Ohmic conduction) Miller and Abrahams have shown that rate equation can be mapped on the impedance network. According to this treatment, the transition rate for the hopping process in the electric field may be given as the product of conductance $G_{i j}$ and voltage $V_{i j}$ between sites $r_{i}$ and $r_{j}$. The conductance $G_{i j}=R_{i j}^{-1}$, linking each pair of sites $(i, j)$ is

$$
R_{i j}^{-1}=\left(e^{2} / k T\right) \gamma_{p h} \exp \left(\frac{\Delta_{i j}}{k T}+2 r_{i j} / a\right)
$$

and the capacitance values $C_{i}$, linking each sites to the external field generator $E_{0} \cos \omega t r_{i}$, are

$$
C_{i}=\left(e^{2} / k T\right) \exp \left(-E_{i / k T}\right)
$$

Here $\gamma_{p h}$ is the phonon frequency of about $10^{12} \mathrm{~Hz}$ and $\Delta_{i j}$ is equal to $\left|E_{i}-E_{j}\right|$ or $\left|E_{j}\right|$ depending on which is greater. All energies are related to the Fermi level $E_{f}$ 


\subsection{Pair approximation}

At high frequency, the two-site model (pair approximation) and its modification have been commonly used $[8,14,16]$. According to these models, the charge hopping takes place between nearest neighbour sites (single hopping) and it is assumed that each individual relaxation process occurs in parallel and independently of all others. The exact form of $\sigma(\omega)$ depends on details of the transition rate relations describing movement of charge carrier between a pair of sites.

In the simplest approximation only sites within $\mathrm{kT}$ of the Fermi level are involved in $\mathrm{AC}$ conduction. It is also assumed that pairs with relaxation time $\tau=1 / \omega$ make the dominant contribution to $\sigma(\omega)[8]$. In order to obtain expression for the $\sigma(\omega)$, the product of the $\mathrm{AC}$ current $J_{i j}$, of the pair i, $j$ and the density of pairs of the length rij must be summed to give

$$
\sigma(\omega)=\sigma_{d c}+\sum_{i, j} n_{i j} J_{i j}
$$

Calculating this expression at any frequency $\omega$ gives for $\sigma(\omega)[17]$

$$
\sigma(\omega)=\sigma_{d c}+0.3 r_{\omega}^{2} a\left[N\left(E_{f}\right) k T\right]^{2} e^{2} \omega / k T
$$

where $r_{\omega} \cong a \ln \left(v_{p h} / \omega\right)$ is greater than the hopping distance. The numerical factor in Eq. (8) differs slightly from the value presented in [15].

At high frequencies, the main results of pair approximation have been confirmed, but at low frequencies discrepancies exist and a modification has been needed [17-19].

\subsection{Cluster approximation}

At low frequencies, during a half period of the oscillation of an external field, the carriers make multiple hopping and the pair approximation fails. Therefore, at low frequency range, hopping charge transport has been formulated in terms of random walk theory of Scher and Lax [12], cluster approach of Bötcher et al. [15] and equivalent random resistor network of Summerfield and Butcher [16]. From this treatment as well as from the computer simulation [20] it follows that, at very low frequencies, the quadratic law $\sigma(\omega) \sim \omega^{2}$ should be fulfilled. However, the experimental results show that the frequency exponent $s$, at very low frequencies, is subquadratic $(1<s<2)$. By application of a low frequency electric field to a disordered system, large clusters of charge carriers with the percolation path of the fractal structure are randomly generated $[19,21]$. At low frequencies, the mechanism of cluster charge transport dominates and individual relaxation process of clusters must be considered. The conductivity $\sigma(\omega)$ can be expressed as the sum of $\sigma_{d c}$ and the cluster polarization conductivity $\sigma_{p}(\omega)$. Since the exact calculation of cluster polarization current are unavailable, therefore the simple approximation has been proposed by Hunt [17-19]. The complexity of large cluster structures composed of many percolation paths has then been replaced by a collection of chains with a resistor oriented in the direction of the applied field. Replacement of clusters by one dimensional chains was proposed by several authors $[22,23]$. The polarization currents $J_{N R}$ of chain composed of $N$ resistors with resistivity $R$ and $N$ 
capacitors with capacitance $C$ may be expressed in the same way as in the pair approximation, if we replace $R$ by $N R, C$ by $N C$ and with $\tau_{N} \propto N^{2} \tau$ to get $[17,18]$

$$
J_{N, R}(\omega)=\left(2 / 3 \pi^{2}\right)\left(N l^{2} / R\right)\left[\omega^{2} \tau_{N}^{2} /\left(1+\omega^{2} \tau_{n}^{2}\right)\right]
$$

The total conductivity is the sum over all contributing chains [17]

$$
\sigma(\omega)=\sigma_{d c}+\Sigma l_{N, R} \cdot n_{N, R}
$$

wheren $n_{N, R}$ is the number of chains.

The statistical distribution function density of chains $n_{N, R}$ on a given cluster has been adopted from the Stauffer formula of cluster distribution in the percolation system [24]. According to this approach, AC conduction at low frequencies may be expressed as [19]

$$
\sigma(\omega)=\sigma_{d c}\left\{1+\left[A \cdot \frac{\omega}{\omega_{c}}\right]^{\frac{d-1}{2}}+\left[A \cdot \frac{\omega}{\omega_{c}}\right]^{\frac{d-1+1 / v}{2}}\right\}
$$

where $A=\left(\frac{T}{T_{0}}\right)^{1 / 4}, d$ is the dimension of conducting system, $v$ is critical exponent of the correlation length $v=0.9[24]$ and $T_{0}=\left[N\left(E_{f}\right) a^{3} k\right]^{-1}$ with $N\left(E_{f}\right)$ being the density of states at the Fermi level and $\omega_{c}$ is the critical frequency corresponding to the onset of dispersion. It is evident that in the case of a 3D system, two different terms, one with linear $s=1$ and the second with a supralinear frequency exponent $1<s<2$, contribute to the total conductivity.

\section{Method and results}

In the experiment, a step voltage $V(t)=V_{0}$ applied to a dielectric sample, generates the charging current $J_{d}$ which is composed of a transient polarization current $J_{p}(t)$ and a steady state conduction current $J_{d c}$. Discharging current $J_{d}$, flowing after the removal of polarization voltage does not involve a steady state component. In the case of low polarization field (linear condition), $J_{d}=-J_{p}(t)$. Details of the experimental procedure and sample characterization are presented in $[25,26]$. The AC conductivity has been calculated by taking the Fourier transformation of the transient currents $J_{p}(t)$. The numerical calculation of the Fourier transformation was based on a simple summation

$$
J(\omega)=\Delta t \sum_{n=0}^{\infty} \exp (-i \omega n \Delta t) \cdot J(n \Delta t)
$$

The summation was carried out at various periods of time with various time intervals $\Delta t$ changing from $\Delta t=1 \mathrm{~s}$ to $\Delta t=1000 \mathrm{~s}$. The charging and discharging currents were measured over a long period of time up to $10^{5} \mathrm{~s}$. The measurements at low temperatures were performed 
down to $77 \mathrm{~K}$ but at higher temperatures the measurements were performed at temperatures which were lower than the Curie temperature. To eliminate the non-linear effects, the samples at low temperature were poling at field $0.2 \mathrm{kV} / \mathrm{cm}$, and at high temperatures, at field $0.02 \mathrm{kV} / \mathrm{cm}$. As an example, in Fig. 1 the time dependent decaying charging $J_{c}(t)$ and discharging currents, $J_{d}(t)$ for sample with $y=0.43$ are shown in $\log$-log scale. The charging currents $J_{c}(t)$ were used to calculate the total AC conductivity $\sigma(\omega)$ which contains the DC component. The discharge (depolarization) current was used to calculate $\sigma(\omega)$. In Figs. 2, 3 and 4, the low frequency dependent AC conductivity for samples with $y=0.43,0.44$ and 0.47 are presented respectively. In table I, the frequency exponent $s$ are listed for the same samples. The power exponents are given for the frequencies above and below the cross-over frequency $\omega_{i}$. It is evident that frequency exponent $s$ depends on the sample composition and temperature. The samples with the morphotropic composition containing less $\mathrm{Ti}$ (with $y=0.43$ and 0.44 ) have a lower $s$ value than those with the non-morphotropic composition (with $y=0.47$ ). For the frequency range $\omega<<\omega_{c}$ the exponents is greater than one, but only slightly in the case of samples with $y=0.43$ and 0.47 .

\section{Discussion}

1. The experimental evidence and theoretical conclusions [27-29] show that the dielectric relaxation and transport properties in perovskite structures are closely related to the oxygen vacancies. The oxygen vacancies are created by losses of oxygen from the crystal lattice during sintering at high temperature according to

$$
\mathrm{O}_{\mathrm{O}} \rightleftarrows V_{\dot{\mathrm{O}}}+1 / 2 \mathrm{O}_{2}
$$

The oxygen vacancies are positively charged with respect to the lattice and always appear single ionized at low temperature $V_{O} \rightleftarrows V_{O}^{0}+e^{\prime}$ or doubly ionized at high temperature $\left(V_{\dot{o}} \rightleftarrows V_{\ddot{O}}+e^{\prime}\right)$. In perovskites, the energy of the first ionization of oxygen vacancies is about $0.1 \mathrm{eV}$, whereas the second energy ionization is about $1.4 \mathrm{eV}$. The thermally liberated electrons contribute to the dielectric relaxation and conduction process. In $\mathrm{Pb}\left[\left(\mathrm{Fe}_{1 / 3} \mathrm{Sb}_{2 / 3}\right)_{x} \mathrm{Ti}_{y} \mathrm{Zr}_{z}\right] \mathrm{O}_{3}$, the $\mathrm{Fe}^{3+}$ ions are incorporated into the perovskite $\mathrm{B}$ site acting as acceptors [27]. At high sintering temperature, the trivalent $\mathrm{Sb}$ ions substitute the divalent $\mathrm{Pb}$ ions and create lead vacancies $V_{P b}$.

$$
\mathrm{Sb}_{2} \mathrm{O}_{3} \rightleftarrows 2 \mathrm{Sb}_{\ddot{P} b}+3 \mathrm{O}_{O}^{x}+V_{P b}^{\prime \prime}
$$

The lead vacancy $V_{P b}^{\prime \prime}$ carries two excess negative charges. The oxygen vacancies existing in perovskite compound migrate around the $\mathrm{Fe}$ ions leading to formation of dipolar defects- $F e_{T i Z r}^{\prime} V_{\ddot{O}}$ complexes. The orientation of these dipolar defects depends on $V_{0}$ location in the oxygen octahedron. A linear dielectric relaxation is connected not only with dipoles but also with potentially mobile charges (electrons, polarons or ions) The observed dielectric relaxation may originate from the $\mathrm{Fe}^{2+} / \mathrm{Fe}^{3+}$ mixed valence structure of 


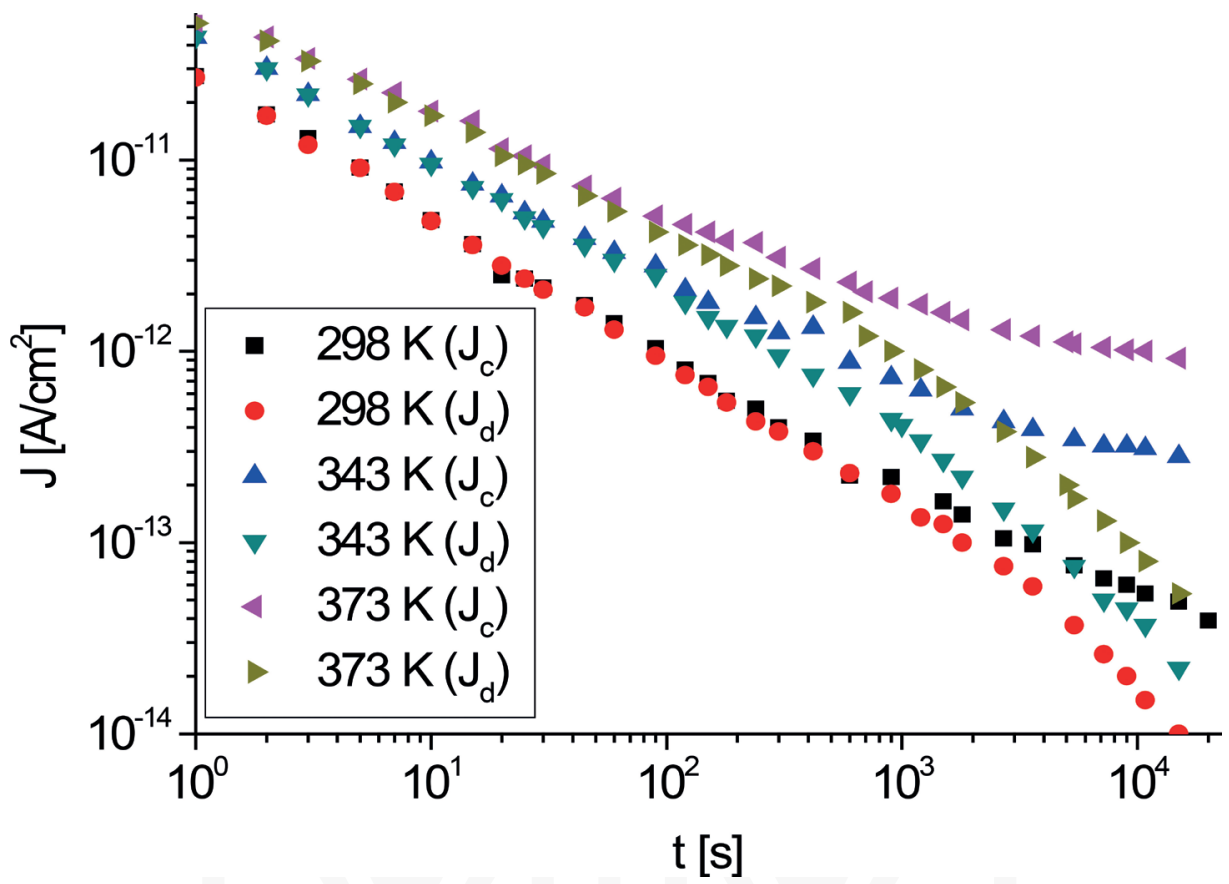

Fig. 1. Time dependence of charging $J_{c}(t)$ and discharging $J_{d}(t)$ currents for sample $y=0.43$ measured at temperatures $298 \mathrm{~K}, 343 \mathrm{~K}$, and $373 \mathrm{~K}$. Poling field: $0.02 \mathrm{kV} / \mathrm{cm}$

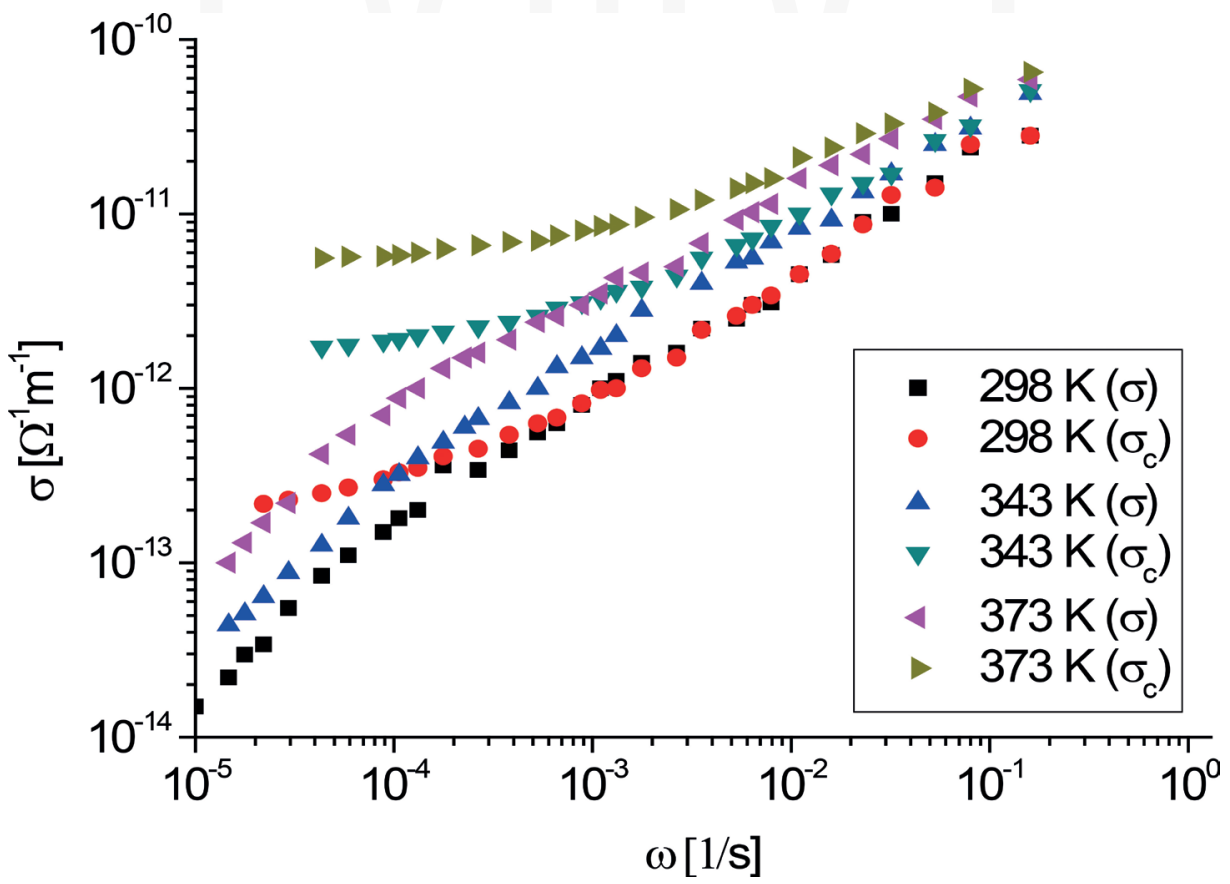

Fig. 2. The low frequency AC conductivity for sample with $y=0.43$ at selected temperatures; $\sigma_{c}(\omega)-$ total AC conductivity $\sigma(\omega)$ - polarization conductivity 

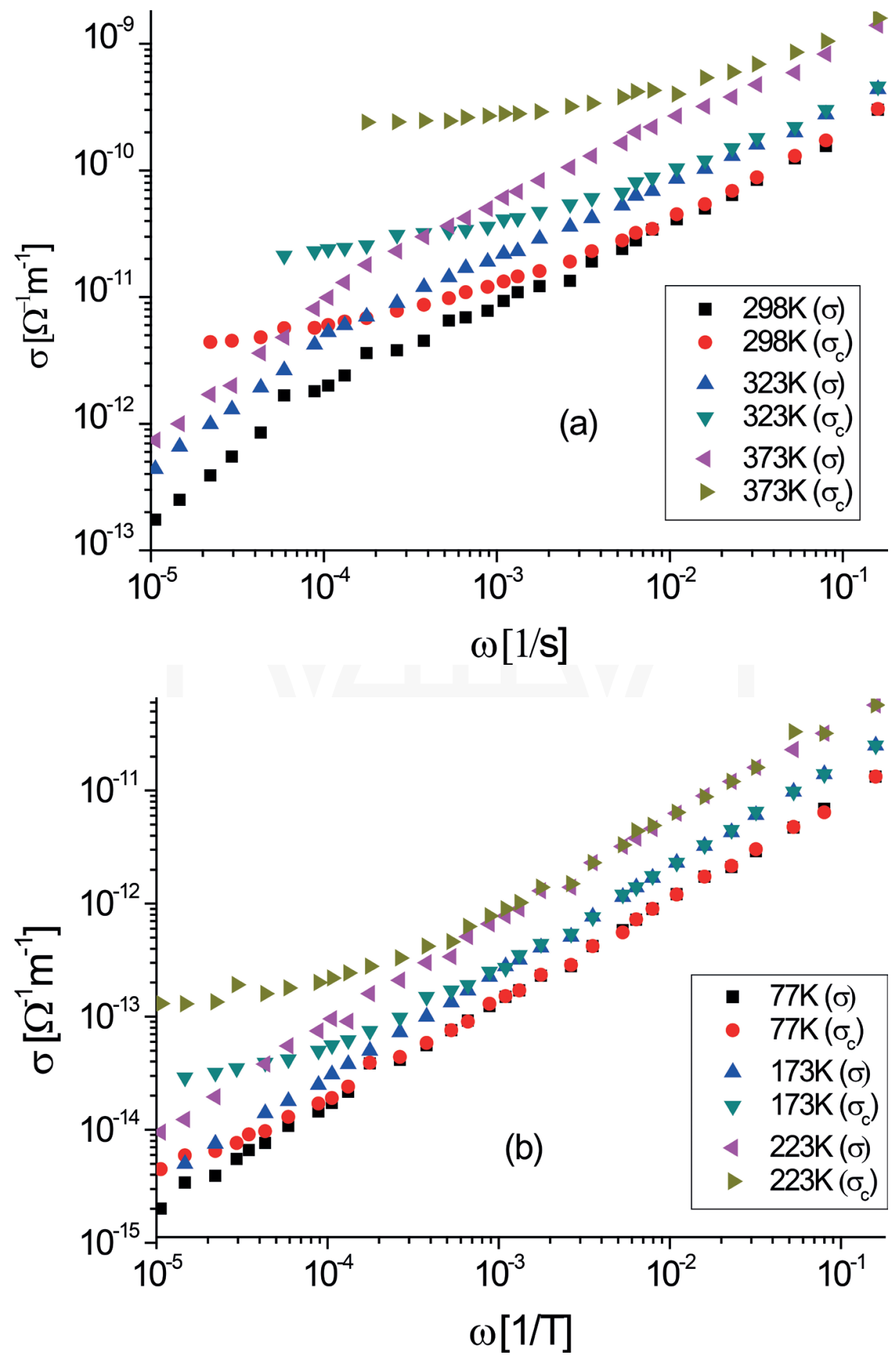

Fig. 3. The low frequency AC conductivity for sample with $y=0.44$ at selected high $(a)$ and low $(b)$ temperatures 

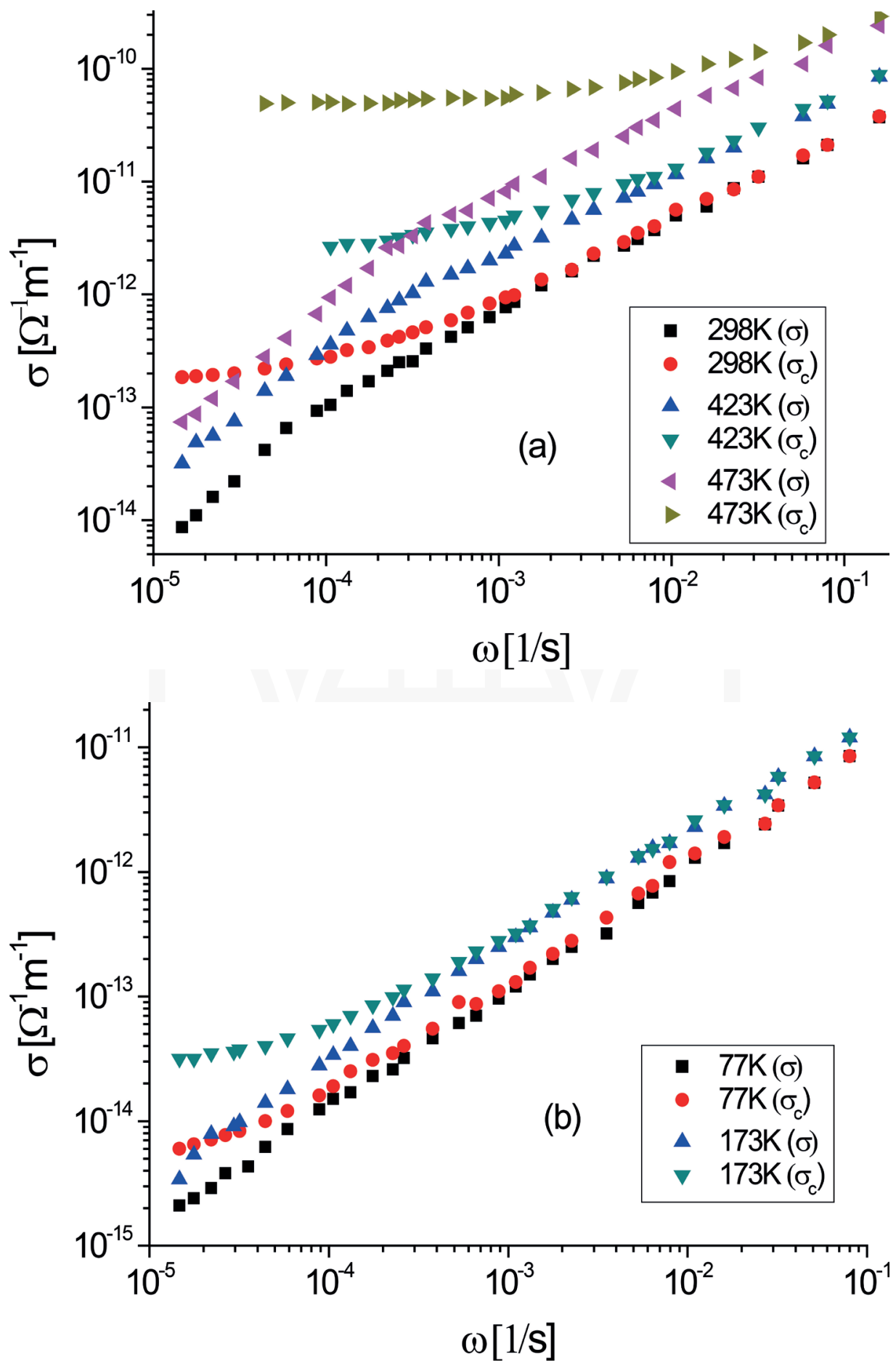

Fig. 4. The low frequency AC conductivity for sample with $y=0.47$ at selected high $(a)$ and low $(b)$ temperatures 
$\mathrm{Pb}\left[\left(\mathrm{Fe}_{1 / 3} \mathrm{Sb}_{2 / 3}\right)_{\mathrm{x}} \mathrm{Ti}_{\mathrm{y}} \mathrm{Zr}_{\mathrm{z}}\right] \mathrm{O}_{3}$. The 3d electrons in $\mathrm{Fe}^{3+}$ may hop to $\mathrm{Fe}^{2+}$ ions; the same hopping movement may take place between $\mathrm{Ti}^{3+} \rightarrow \mathrm{Ti}^{2+}$ and $\mathrm{Zr}^{3+} \rightarrow \mathrm{Zr}^{2+}$ ions. This electron hopping creates time dependent dipole moments. At higher frequencies, the carrier hopping takes place between sites separated by lower energy, whereas at lower frequencies, the energy barrier with higher energy must be over-passed. This way, the AC conductivity at higher frequencies is greater than at lower ones. At higher temperatures, the energy distribution of barriers becomes more uniform and variation of AC conductivity with frequency becomes weaker. The dielectric relaxation can be also attributed to the motion of dipoles created by the defect associate $\left(F e_{T Z Z r}^{\prime} V_{\ddot{O}}\right)$ under the action of an external field. In the electric field, the defect dipole can be reoriented only slightly in the field direction or may perform greater movements. In the latter case, changes in position of $V_{\ddot{o}}$ within the oxygen octahedron via hopping process will result in reorientation of the complex defect dipole. The relaxation time in this case is determined by thermally activated diffusive jumping of the oxygen vacancies. The long range movement of weakly bonded electrons gives rise to the $\mathrm{DC}$ conduction that can be described by the hopping mechanism. The transport of electrons from cathode to anode, contributing to the DC conduction, needs to overcome a higher potential barrier, as compared to a lower energy barrier needed to overcome a shorter displacement in the case $\mathrm{AC}$ conduction. Therefore the activation energy of DC conductivity is higher than the activation energy of AC conductivity.

2. The temperature dependence of the frequency exponent $s(T)$ is consistent with results of multiple hopping models $[13,20,21]$. According to these models, $s$ should decrease with increasing temperature, as can be seen from results presented in Table I. The exponent $s$ depends also on concentration $N$ of localization sites. The concentration $N=2.9 \cdot 10^{23} \mathrm{~m}^{-3}$ for sample with $y=0.44$ is greater than concentration $N=1.5 \cdot 10^{23} \mathrm{~m}^{-3}$ for sample with $y=0.47$ [25]. Indeed, for all temperatures, the exponent s for sample with $y=0.44$ is smaller than that for sample with $y=0.47$. Moreover, the values of s are much smaller than 2 , being at variance with the prediction of the cluster model [10] and computer simulation [20] yielding $s=2$. It is evident from expression (11) that two different terms for low $\omega$ exist: one linear and the second supralinear. For three dimensional systems, $d=3$ and $v=0.9$ [24] and the frequency exponent equals $(d-1+1 / v) / 2=1.55$.

The results presented in Table I show that the frequency exponent for all samples is less than the theoretical value 1.55. These results may suggest that the Debye-type relaxation effects play an important role. In the low temperature range, concentration of charge carriers decreases and contribution of dipolar dielectric relaxation becomes more important.

\section{Conclusions}

1. The presented results suggest that the frequency dependence of $\mathrm{AC}$ conductivity of PZT-PFS at very low frequencies $\sigma(\omega) \sim \omega^{s}$ may be explained in terms of fractal structure of the randomly generated clusters formed by sequentially correlated hopping paths of charge carriers (i. e. the geometry of multiple hopping). 
2. Approximation of cluster current as the one-dimension collection of chain currents leads to low frequency $\mathrm{AC}$ conductivity expression with the frequency exponent $1<s<2$, in accordance with the experimental results.

Table 1. Values of frequency power exponents s for samples with $y=0.43, y=0.44$ and $y=0.47$.

\begin{tabular}{|c|c|c|c|}
\hline$y$ & $T[\mathrm{~K}]$ & $s\left(\omega<\omega_{c}\right)$ & $s\left(\omega>\omega_{c}\right)$ \\
\hline \multirow{4}{*}{$y=0.43$} & 298 & 1.38 & 0.62 \\
\cline { 2 - 4 } & 343 & 1.35 & 0.62 \\
\cline { 2 - 4 } & 373 & 1.38 & 0.577 \\
\hline \multirow{5}{*}{$y=0.44$} & 298 & 1.37 & 0.62 \\
\cline { 2 - 4 } & 323 & 1.41 & 0.58 \\
\cline { 2 - 4 } & 373 & 1.47 & 0.577 \\
\cline { 2 - 4 } & 223 & 1.36 & 0.84 \\
\cline { 2 - 4 } & 173 & 1.31 & 0.43 \\
\cline { 2 - 4 } & 77 & 1.27 & 0.81 \\
\hline \multirow{5}{*}{$y=0.47$} & 298 & 1.33 & 0.81 \\
\cline { 2 - 4 } & 423 & 1.31 & 0.70 \\
\cline { 2 - 4 } & 473 & 1.34 & 0.675 \\
\cline { 2 - 4 } & 173 & 1.42 & 0.81 \\
\cline { 2 - 4 } & 77 & 1.38 & 0.91 \\
\hline
\end{tabular}

The author is grateful to Prof. J. Cisowski and Ph.D. W. Osak for suggestions and helpful discussion.

\section{References}

[1] Shirane G., Suzuki K., Takeda A., Phase transition in Solid Solutions $\mathrm{PbZrO}_{3}$ and $\mathrm{PbTiO}_{3}$ (II) X ray study, Journal of the Physical Society of Japan, Vol. 7, 1952, 12-18.

[2] Jaffe B., Cook W.R., Piezoelectric Ceramic, Academic Press, London 1971, 136.

[3] Noheda B., Cox D.E., Gonzalo J.A., Cross L.E., Park S.E., A monoclinic ferroelectric phase in the $\mathrm{Pb}$ (Zr1-xTix) $\mathrm{O}_{3}$ solid solution, Applied Physics Letters, Vol. 74, 1999.

[4] Glazer A.M., Thomas P.A., Baba-Kishi K.Z., Pang G.K.H., Tai C.W., Influence of short-range and long range order on the evolution of the morphotropic phase boundary of $\mathrm{Pb}\left(\mathrm{Zr}_{1-x} \mathrm{Ti}_{x}\right) \mathrm{O}_{3}$, Physical Reviev B, Vol. 70, 184123-1-9.

[5] Osak A., Pawelczyk M., Ptak W.S., Investigation of the structure, pyro- and piezoelectric properties of a ferroelectric ceramics of PZT+(FeSb), Ferroelectics, Vol. 186, 1996, $123-126$. 
[6] Elliot S. R., Ac conduction in amorphous chalcogenite and pnictidesemicondactors, Advances in Physics, Vol. 36, 1987,131-218.

[7] Jonsher A.K., Dielectric relaxation in solids Ch. 4, Chelsae Dielectric Press, London 1984.

[8] Pollak M. On the Frequency Dependence of Conductivity in Amorphous Solids, Philosophical Magazine, Vol. 23, 1971, 519-2.

[9] Miller A., Abrahams E., Impurity Conduction at Low Concentration, Physical Review, Vol. 120, 1960, 745-755.

[10] Ambegaokar V., Halperin B.J., Langer J.S., Hopping Conductivity in Disordered Systems, Physical Review B, Vol. 7, 1971, 2612-2620.

[11] Butcher P.N., On the rate equation formulation of the hopping conductivity problem, Journal of Physics C: Solid State Physics, Vol. 5, 1972, 1817-1829.

[12] Scher H., Lax M., Stochastic transport in disordered solids, Physical Review B, Vol. 7, 1973, 4491-4519.

[13] Dyre J.C., The radom free-energy barier model for AC conduction in disordered solids, Journal of Applied Physics, Vol. 64, 1988, 2456-2468.

[14] Pike G.E., Ac conductivity of scandium oxide and new hopping model of conductivity, Physical Review B, Vol. 6, 1972, 1572-1580.

[15] Böttger H.B., Bryksin V.V., Yashin G. Yu., Cluster approximatelly in the theorry of hopping model of conductivity in disordered solids, Journal Physics C: Solids State Physics, Vol. 12, 1979, 2797-2808.

[16] Summerfield S., Butcher P.N., A unified equivalent-circuit approach to the theory of AC and DC hopping conductivity in disordered systems, Journal of Physics C: Solid State Physics, Vol. 15, 1982, 7003-7016.

[17] Hunt A., The AC conductivity of variable range hopping systems such as amorphous semiconductors, Philosophical Magazine B, Vol. 64, 1991, 579-589.

[18] Hunt A., The AC conductivity of the Fermi glass. A model for glassy conduction, Solid State Communication, Vol. 80, 1991, 151-155.

[19] Hunt A., Frequency dependent conductivity of the Fermi glass, Journal of Physics Condensed Matter, Vol. 4, 1992, 6957-6970.

[20] Moore E.J., Numerical studies of the AC conductivity of hopping system I. Effects of space and energy disorder, Journal of Physics C: Solid State Physics, Vol. 7, 1974, 1840-1853.

[21] Niklassan G.A., Fractal aspects of the dielectric response of charge carriers in disordered materials, Journals of Applied Physics, Vol. 62, 1987, R1-14.

[22] Pollak M., Pohl H.A., Dielectric dispersion in some polymer and polyelectrolyte. A model, Journal Chemical Physics, Vol. 67, No. 7, 1975, 2980-2987.

[23] Skal A.S., Shklovski B.J., Soviet Physics of Semiconductors, Vol. 8, 1975, 1029.

[24] Stauffler D., Introduction to Percolation Theory, Taylor and Francis, London 1985.

[25] Osak A., Jankowska-Summara I., Electrical transport in ferroelectric $\mathrm{Pb}\left[\left(\mathrm{Fe}_{1 / 3} \mathrm{Sb}_{2 / 3}\right)_{x} \mathrm{Ti}_{y} \mathrm{Zr}_{z}\right] \mathrm{O}_{3}$ ceramics, Phase Transitions, Vol. 82, 2009, 899-909.

[26] Osak A., Ultra low frequency dielectric dispersion in PZT-PFS ferroelectric ceramics, Journal of Advanced Dielectrics, Vol. 3, 2013. 
[27] Erdem E., Eichel R.A., Kungl H., Hoffman M.J., Ozarowski A., van Tal J., Brunel L.C., Characterization of $\left(\mathrm{Fe}_{\mathrm{ZrTi}}{ }^{-V_{O}^{\prime \prime}}\right)$ defects dipoles in ( $\left.\mathrm{La}, \mathrm{Fe}\right)$ codoped PZT 52.2/47.5 Piezoelectric ceramics by Multifrequency Electron Paramagnetic Resonance Spectroscopy, IEEE Trans. Ultrasonic, Ferroelectrics, Frequency Contr. Vol. 55, 2008, 1061-1068.

[28] Chen Ang., Zhi Yu and Cross L.E., Oxygen-vacancy low-frequency dielectric relaxation and electrical conduction in Bi:SrTiO, Physical Review B, Vol. 62, 2000, 228-236.

[29] Marton P., Elsässer C., Switching of a substitutional-iron/oxygen-vacancy defect complex in ferroelectric $\mathrm{PbTiO}_{3}$ from first principles, Physical Review B, Vol. 83, 2011, 020106-1-4. 